\title{
ON THE INJECTIVE HULLS OF SEMISIMPLE MODULES
}

\author{
BY \\ JEFFREY LEVINE( ${ }^{(1)}$
}

\begin{abstract}
Let $R$ be a ring. Let $T=\bigoplus_{i \in I} E\left(R / M_{i}\right)$ and $W=\prod_{i \in I} E\left(R / M_{i}\right)$, where each $M_{i}$ is a maximal right ideal and $E(A)$ is the injective hull of $A$ for any $R$-module $A$. We show the following: If $R$ is (von Neumann) regular, $E(T)=T$ iff $\left\{R / M_{i}\right\}_{i \in I}$ contains only a finite number of nonisomorphic simple modules, each of which occurs only a finite number of times, or if it occurs an infinite number of times, it is finite dimensional over its endomorphism ring.

Let $R$ be a ring such that every cyclic $R$-module contains a simple. Let $\left\{R / M_{i}\right\}_{i \in I}$ be a family of pairwise nonisomorphic simples. Then $E\left(\oplus_{i \in I} E\left(R / M_{i}\right)\right)=\prod_{i \in I} E\left(R / M_{i}\right)$. In the commutative regular case these conditions are equivalent.

Let $R$ be a commutative ring. Then every intersection of maximal ideals can be written as an irredundant intersection of maximal ideals iff every cyclic of the form $R / \bigcap_{i \in I} M_{i}$, where $\left\{M_{i}\right\}_{i \in I}$ is any collection of maximal ideals, contains a simple.

We finally look at the relationship between a regular ring $R$ with central idempotents and the Zariski topology on spec $R$.
\end{abstract}

Introduction. Eckmann and Schopf [2] have shown the existence and uniqueness up to isomorphism of the injective hull of any module. The problem has been to take a specific module and find explicitly its injective hull. In this paper we will do this for certain semisimple modules.

A. K. Tiwary [13] has a description of the injective hull of any semisimple module over a commutative regular ring in terms of the Zariski topology.

Let $R$ be a ring and $S$ a semisimple $R$-module. Then $S \approx \oplus_{i \in I} S_{i}$, where each $S_{i}$ is a simple $R$-module. Let $T=\bigoplus_{i \in I} E\left(S_{i}\right)$ and $W=\prod_{i \in I} E\left(S_{i}\right)$, where $E(M)$ is the injective hull of $M$ for any $R$-module $M$. In $\S 2$ we give necessary and sufficient conditions for $E(S)=T$, when $R$ is a regular ring. In $\S 3$ sufficient conditions are given for $E(S)=W$ and these are shown to be necessary when $R$ is a commutative regular ring.

A closely related problem is the following: When can an intersection of maximal right ideals be written as an irredundant intersection of maximal right ideals?

Received by the editors June 22, 1970.

AMS 1970 subject classifications. Primary 16A30, 16A52; Secondary 16A66, 54A20, 54H10.

Key words and phrases. Regular, direct sum of modules, direct product of modules, injective, Zariski topology, semisimple.

$\left.{ }^{1}\right)$ This research was partially supported by a National Defense Title IV Fellowship and a grant from the Grant-in-Aid Committee of Monmouth College.

Copyright (C) 1971, American Mathematical Society 
Nastasescu and Popescu [8] have shown that in a commutative ring $R$ a sufficient condition is that every cyclic $R$-module contains a simple $R$-module. This condition is too strong to be necessary.

In $\$ 4$ we give a necessary and sufficient condition that every intersection of maximal ideals can be written as an irredundant intersection of maximal ideals in a commutative ring.

$\S 5$ deals with some of the properties of the Zariski topology on spec $R$ of a regular ring whose idempotents are central. Alexandroff and Urysohn [1] have shown that the Stone-Čech compactification of a set $I$ with the discrete topology has the property that the only converging sequences are those that are constant from some point on. Using the fact that this topology is homeomorphic to the Zariski topology on any direct product of division rings indexed by $I$ [6], [11], we give an algebraic proof of this property. In fact, we show that every complete regular ring with central idempotents has this property. We also look at the closed sets and how they relate to the preceding sections.

This paper is part of a doctoral dissertation submitted to Rutgers University. The author wishes to express his appreciation to his advisor Professor Barbara Osofsky for her encouragement and advice during the writing of this thesis.

1. Conventions and definitions. Throughout this paper $R$ will denote an associative ring with unit. Unless explicitly mentioned to the contrary all $R$-modules will be unital right $R$-modules.

A ring $R$ is called (von Neumann) regular iff every finitely generated right ideal is generated by an idempotent. A ring $R$ is called a $V$-ring iff every simple $R$ module is injective. S. Villamayor has shown that $R$ is a $V$-ring iff every right ideal is an intersection of maximal right ideals. This result is unpublished. If $R$ is commutative, $R$ is a $V$-ring iff $R$ is regular [10]. Let $I$ be any set. Then by $|I|$ we mean the cardinality of $I$. By $M^{(I)}$ we mean the direct sum of the $R$-module $M$ indexed by $I$. By $M^{I}$ we mean the direct product of $M$ indexed by $I$.

Let $R$ be a ring and let $M \in M_{R}$ have endomorphism ring $\Lambda$. For any subset $x$ of $M$, let $x^{\perp}=\{r \in R \mid x r=0\} . x^{\perp}$ is a right ideal of $R$. The totality of such right ideals will be denoted by $A_{r}(M, R)$. For any subject $Y$ of $R$, let $Y^{\perp}=\{m \in M \mid m Y=0\}$. Then $Y^{\perp}$ is a $\Lambda$-submodule of $M$. The totality of such $\Lambda$-submodules of $M$ is denoted by $A_{l}(M, R)$. It is clear that $A_{r}(M, R)$ (respectively $A_{l}(M, R)$ ) is closed under arbitrary intersections, making it a complete lattice. In particular the ascending chain condition (a.c.c.) in $A_{r}(M, R)$ (respectively $A_{l}(M, R)$ ) is equivalent to the maximum condition. Since $A_{r}(M, R)$ and $A_{l}(M, R)$ are anti-isomorphic as lattices, one will satisfy a.c.c. iff the other satisfies the descending chain condition (d.c.c.).

A module $M$ is said to be $\Sigma$-injective iff $M^{(I)}$ is injective for any index set $I . M$ is said to be countably $\Sigma$-injective in case $M^{(\omega)}$ is injective. $M$ is $\Sigma$-injective iff $M$ is countably $\Sigma$-injective [3, Proposition 3]. 
2. The direct sum as injective hull. Let $R$ be a ring. Let $S$ be a semisimple $R$-module. Then $S \approx \bigoplus_{i \in I} S_{i}$ where each $S_{i}$ is a simple $R$-module. Let

$$
T=\bigoplus_{i \in I} E\left(S_{i}\right) \text { and } W=\prod_{i \in I} E\left(S_{i}\right)
$$

$W$ is injective and it is trivial that $T$ is an essential extension of $S$. Therefore, an isomorphic copy of $E(S)$ can be found contained in $W$ and containing $T$. Thus $E(S)=E(T)$. We will give necessary and sufficient conditions for $E(T)=T$ in the case of a regular ring.

LeMma 2.1. Let $R$ be a ring. Let $A=\bigoplus_{i \in I} A_{i}$, where each $A_{i}$ is an $R$-module, and $|I|$ is infinite. Let

$$
0 \neq x=\left(x_{i}\right)_{i \in I} \in \prod_{i \in I} A_{i}
$$

such that $x R \cap A=0$. Let $r \in R$ such that $r \notin\left(X_{j}\right)^{\perp}$ for some $j \in I$. Then

$$
I^{\prime}=\left\{i \in I \mid x_{i} r \neq 0\right\}
$$

is infinite.

Proof. If not, then $0 \neq\left(x_{i} r\right)_{i \in I} \in x R \cap A$. This contradicts the hypothesis on $x$. Therefore $I$ is infinite.

LEMMA 2.2. Let $R$ be a ring. Let $M_{1}$ and $M_{2}$ be maximal right ideals, such that $R / M_{1} \not R / M_{2}$. Let $0 \neq r+M_{1} \in R / M_{1}$ and $0 \neq s+M_{2} \in R / M_{2}$. Then $\left(r+M_{1}\right)^{\perp}$ $\neq\left(s+M_{2}\right)^{\perp}$.

Proof. Since $R / M_{1}=\left(r+M_{1}\right) R \approx R /\left(r+M_{1}\right)^{\perp}$ and $R / M_{2}=\left(s+M_{2}\right) R \approx R /\left(s+M_{2}\right)^{\perp}$, if $\left(s+M_{2}\right)^{\perp}=\left(r+M_{1}\right)^{\perp}$, then $R / M_{1} \approx R / M_{2}$. Thus the conclusion holds.

$T$ will not be injective if we can find elements in $\prod_{i \in I} E\left(R / M_{i}\right)$ which are essential over their intersection with $\bigoplus_{i \in I} E\left(R / M_{i}\right)$. The following technical lemma will construct these elements.

LEMMA 2.3. Let $R$ be a regular ring. Let $T=\oplus_{i \in I} E\left(R / M_{i}\right)$, where each $M_{i}$ is a maximal right ideal and $|I|$ is infinite. Let $0 \neq x=\left(x_{i}\right)_{i \in I} \in \prod_{i \in I} R / M_{i}$ such that $x R \cap\left(\oplus_{i \in I} R / M_{i}\right)=0$. Let $H=\left\{i \in I \mid x_{i} \neq 0\right\}$. Pick $a \in H$. Assume

(i) $\left(X_{h}\right)^{\perp} \neq\left(X_{h^{\prime}}\right)^{\perp}$ for all $h, h^{\prime} \in H, h \neq h^{\prime}$.

(ii) For all $e=e^{2} \in R$ with $e \in\left(X_{a}\right)^{\perp}$, if there exists $a b \in H$ such that $e \notin\left(X_{b}\right)^{\perp}$, then there exists an infinite set $H(e) \subseteq H$ such that $(1-e) \notin\left(X_{h}\right)^{\perp}$ and $\left(X_{a}\right)^{\perp}$ $\neq\left(1-e+\left(X_{h}\right)^{\perp}\right)^{\perp}$ for all $h \in H(e)$.

Then $T$ is not injective.

Proof. We will construct a countable set $J \subseteq H$, a set of idempotents $\left\{e_{j}\right\}_{j \in J}$ and a set of nonzero $R$-maps $\left\{g_{j}\right\}_{j \in J}$ such that $\sum_{j \in J} e_{j} R$ is direct and $g_{j}: e_{j} R \rightarrow E\left(R / M_{j}\right)$ for all $j \in J$. 
If this is done, let $g$ be the direct sum map from $\bigoplus_{j \in J} e_{j} R$ to $T$. Since the image of $g$ has an infinite number of nonzero projections in $T$, there does not exist an $m \in T$ such that $g(x)=m x$ for all $x \in \bigoplus_{j \in J} e_{j} R$. Therefore, $T$ is not injective.

We now proceed to the construction. It will be based on an induction process. For each integer $n$ we will construct the following sets.

$A_{n}=\left\{e_{1}, \ldots, e_{n}\right\}$ of $n$ distinct idempotents such that $e_{i} \in\left(X_{a}\right)^{\perp}$ for $1 \leqq i \leqq n$ and $\sum_{i=1}^{n} e_{i} R$ is direct. Also $A_{i}=A_{i-1} \cup\left\{e_{i}\right\}$ for all $i \leqq n$.

$L_{n}=\left\{M_{p_{n}}(i) \mid 1 \leqq i \leqq n\right\}$ where $p_{n}(i)$ is a one-to-one map from $\{1,2, \ldots, n\} \rightarrow H$ such that $p_{n}$ extends $p_{n-1}$.

Each $e_{i} \notin M_{p_{n}}(i)$ and $L_{n}=L_{n-1} \cup\left\{M_{p_{n}}(n)\right\}$.

Let $b \in H, b \neq a$. Let $e_{1}=e_{1}^{2} \in\left(X_{a}\right)^{\perp}, e_{1} \notin\left(X_{b}\right)^{\perp}$. Let $A_{1}=\left\{e_{1}\right\}$ and $L_{1}=\left\{M_{b}\right\}$.

Assume we have $A_{i}$ and $L_{i}$ for all $i \leqq n-1$. Let $f_{n}=f_{n}^{2}$ generate $\bigoplus_{i=1}^{n-1} e_{i} R$. Then $\left(1-f_{n}\right)+\left(X_{a}\right)^{\perp}=1+X_{a}^{\perp}$ since $f_{n} \in\left(X_{a}\right)^{\perp}$. Therefore $1-f_{n} \notin\left(X_{a}\right)^{\perp}$ and by Lemma $2.1,1-f_{n}$ does not belong to an infinite subset of $\left\{\left(X_{h}\right)^{\perp}\right\} h \in H$. Therefore by the hypothesis, there exists a set $H\left(f_{n}\right) \subseteq H$ such that $\left|H\left(f_{n}\right)\right|$ is infinite and $\left(X_{a}\right)^{\perp}$ $\neq\left(1-f_{n}+\left(X_{h}\right)^{\perp}\right)^{\perp}$ for all $h \in H\left(f_{n}\right)$. Pick an $h(n) \in H\left(f_{n}\right)$ such that $h(n)$ does not belong to the image of $P_{n-1}$. Then $\left(1-f_{n}\right)+\left(X_{a}\right)^{\perp} \neq 0$ and $\left(1-f_{n}\right)+\left(X_{h(n)}\right)^{\perp} \neq 0$. Since $\left(X_{a}\right)^{\perp} \neq\left(1-f_{n}+\left(X_{h(n)}\right)^{\perp}\right)^{\perp}$, we may pick a $t_{n} \in\left(X_{a}\right)^{\perp}, t_{n} \notin\left(1-f_{n}+\left(X_{h(n)}\right)^{\perp}\right)^{\perp}$. Then $\left(1-f_{n}\right) t_{n} \in\left(X_{a}\right)^{\perp}$ but $\left(1-f_{n}\right) t_{n} \notin\left(X_{h(n)}\right)^{\perp}$. Let $e_{n}=e_{n}^{2}$ generate $\left(\left(1-f_{n}\right) t_{n}\right) R$. Let $A_{n}=A_{n-1} \cup\left\{e_{n}\right\}$ and $L_{n}=L_{n-1} \cup\left\{M_{h(n)}\right\}$. These sets have the required properties.

To get the set of maps $\left\{\boldsymbol{g}_{j}\right\}_{j \in J}$, we use the fact that

$$
R / M_{h(n)} \approx R /\left(X_{h(n)}\right)^{\perp}
$$

Define $g_{n}: e_{n} R \rightarrow R /\left(M_{h(n)}\right)^{\perp}$, by $g_{n}\left(e_{n} r\right)=e_{n} r+\left(X_{h(n)}\right)^{\perp}$ for all $r \in R$. Since $e_{n} \notin\left(X_{h(n)}\right)^{\perp}, g$ is a nonzero $R$-map which cannot be extended to a map from $R$ to $T$.

Proposition 2.4. Let $R$ be a regular ring. Let $T=\bigoplus_{i \in I} E\left(R / M_{i}\right)$, where each $M_{i}$ is a maximal right ideal. If $T=E(T)$, then the set $D=\left\{R / M_{i}\right\}_{i \in I}$ contains only a finite number of nonisomorphic simple modules.

Proof. Assume $D$ contains an infinite number of nonisomorphic simple modules. Pick out a countable subset of nonisomorphic simples, say $\left\{R / M_{b}\right\} b \in \omega$.

If $T$ is injective, so is $T^{\prime}=\bigoplus_{b \in \omega} E\left(R / M_{b}\right), T^{\prime}$ being a direct summand of $T$. Let $0 \neq x=\left(x_{b}\right)_{b \in \omega} \in \prod_{b \in \omega} R / M_{b}$ such that $x R \cap \bigoplus_{b \in \omega} R / M_{b}=0$. If no such element $x$ existed, $T$ would be contained in a proper essential extension and thus $T$ would not be injective.

We claim that $\left(X_{b}\right)^{\perp} \neq\left(X_{b^{\prime}}\right)^{\perp}$ for all $b, b^{\prime} \in \omega$, with $b \neq b^{\prime}$ and $x_{b} \neq 0$ and $x_{b^{\prime}} \neq 0$. This follows since $R / M_{b} \approx R /\left(x_{b}\right)^{\perp}$ and $R / M_{b^{\prime}} \approx R /\left(x_{b^{\prime}}\right)^{\perp}$ and $R / M_{b} \not 2 R / M_{b^{\prime}}$. Let $H=\left\{b \in \omega \mid x_{b} \neq 0\right\}$. Let $e$ be any idempotent in $\left(x_{1}\right)^{\perp}, e \notin\left(x_{2}\right)^{\perp}$. Then there exists an infinite set $H(e) \subseteq H$ such that $\left(1-e+\left(x_{h}\right)^{\perp}\right)^{\perp} \ddagger\left(x_{1}\right)^{\perp}$ for all $h \in H(e)$. Otherwise $R / M_{1} \approx R / M_{b}$ for an infinite number of $b \in \omega$. Now apply Lemma 2.3. Therefore $T$ is not injective. 
The converse of Proposition 2.4 is false. The full ring of linear transformations $R$ on a countable dimensional right vector space $V$ over a field $F$ provides a counterexample. The socle of $R$ is a countable direct sum of isomorphic injective simple $R$-modules, but is not itself injective.

Let $R$ be a ring and let $M$ be a maximal right ideal. Let $F=\operatorname{Hom}_{R}(R / M, R / M)$. Then $F$ is a division ring and $R / M$ is a left vector space over $F$. In the above example each simple module contained in the socle of $R$ was an infinite-dimensional left vector space over its endomorphism ring. If $R$ is regular, the following theorem shows that $R / M$ is $\Sigma$-injective iff $R / M$ is finite dimensional over $F$.

THEOREM 2.5. Let $R$ be a regular ring. Let $R / M$ be a simple $R$-module, where $M$ is a maximal right ideal. Let $F=\operatorname{Hom}_{R}(R / M, R / M)$. Then $E(R / M)$ is $\Sigma$-injective iff $R / M$ is finite dimensional over $F$. In this case $E(R / M)=R / M$.

Proof. Assume $R / M$ is finite dimensional over $F$. Let $\left(x_{i}\right)_{i=1}^{n}$ be a basis and let $M_{i}=\left(x_{i}\right)^{\perp}$. Let $N=\bigcap_{i=1}^{n} M_{i}$. Then $N$ is a two sided ideal, since $N=(R / M)^{\perp} . R / N$ is semisimple since $R / N$ is regular and has a composition series. Let $f: I \rightarrow(R / M)^{(\omega)}$ be an $R$-map, where $I$ is any right ideal. We will show that $f$ can be extended to an $R$-map. $f^{\prime}: R \rightarrow(R / M)^{(\omega)}$, thus showing $(R / M)^{(\omega)}$ is injective by Baer's Criterion.

Let $A=I+N / N$. Then $A$ is a right $R / N$-module and $A \approx I / I \cap N$. Define a map $f^{*}: A \rightarrow(R / M)^{(\omega)}$ by $f(i+(I \cap N))=f(i)$ for all $i \in I$. This is well defined since if $i+(I \cap N)=i^{\prime}+(I \cap N)$, then $i^{\prime}-i \in I \cap N=I \cdot N$ since $R$ is regular [7, p. 133]. Therefore $f\left(i-i^{\prime}\right)=\sum_{i=1}^{n} f\left(a_{i} b_{i}\right)$ where $a_{i} \in I$ and $b_{i} \in N$. Then $\sum_{i=1}^{n} f\left(a_{i} b_{i}\right)$ $=\sum_{i=1}^{n}\left(f\left(a_{i}\right)\right) b_{i}=0$ since $b_{i} \in(R / M)^{\perp}$. Thus $f(i)=f\left(i^{\prime}\right)$. It is easy to see that $f^{*}$ is an $R / N$ homomorphism. Since $(R / M)^{(\omega)}$ is an injective $R / N$ module, $f^{*}$ can be extended to an $R / N$-map, $f^{\#}$, from $R / N$ to $(R / M)^{(\omega)}$. Define $f^{\prime}: R \rightarrow(R / M)^{(\omega)}$ by $f^{\prime}(r)=f(r+N)$ for all $r \in R$. Then $f^{\prime}$ is an $R$-map extending $f$ and $R / M$ is $\Sigma$-injective.

Now assume that $R / M$ is infinite dimensional over $F$. Let $\left\{x_{i}\right\}_{i \in I}$ be a basis. We will show that the hypothesis of Lemma 2.3 holds. Thus $E(R / M)$ is not $\Sigma$-injective.

Let $M_{i}=\left(x_{i}\right)^{\perp}$ for all $i \in I$ and let $L=\bigcap_{i \in I} M_{i}$. Then $L=(R / M)^{\perp}$. Let $\mathscr{D}$ be the set of all right ideals $C$ in $A_{r}(R / M, R)$ such that $C / L$ has a composition series. $\mathscr{D} \neq \varnothing$ since $L \in \mathscr{D}$. If $E(R / M)$ is $\Sigma$-injective, Faith has shown $A_{r}(E(R / M), R)$ [3, Proposition 3] has a.c.c. Let $K$ be maximal in $\mathscr{D}$. Let $\mathcal{O}$ be an $F$-submodule of $R / M$ such that $(\mathcal{O})^{\perp}=K$. Let $\left\{y_{j}\right\}_{j \in J}$ be a basis for $\mathcal{O}$ and let $N_{j}=\left(y_{j}\right)^{\perp}$ for all $j \in J$. Then $K=\bigcap_{j \in J} N_{j}$. We claim $|J|$ is infinite. If not, then $R / K$ has a composition series and therefore $R / L$ would have a composition series.

Let $x_{i 1}, \ldots, x_{i n} \ldots$ be a countable subset of $\left\{x_{i}\right\}_{i \in I}$ where $x_{i j} \neq x_{i k}$ if $j \neq k$. Then

$$
F x_{i 1} \subset_{+} F x_{i 1}+F x_{i 2} \subset_{+} \cdots \subset_{+} F x_{i 1}+\cdots+F x_{i n} \subset_{+} \cdots
$$

and each $F x_{i 1}+\cdots+F x_{i j} \in A_{l}(R / M, R)$ since every simple module is quasiinjective [4]. Since $A_{l}(R / M, R)$ does not satisfy a.c.c., $A_{r}(R / M, R)$ does not satisfy d.c.c. Thus $R / L$ has no composition series. 
Let $y=\left(y_{j}\right)_{j \in J} \in(R / M)^{J}$. Then $y R \cap(R / M)^{(J)}=0$. If not, there would exist an $r \in R, r \in\left(y_{j}\right)^{\perp}$ for all but a finite number of $j \in J$. Let $J^{\prime}=\left(j \mid r \in\left(y_{j}\right)^{\perp}\right)$. Then $P=\bigcap_{j \in J^{\prime}} N_{j} \supset_{+} \bigcap_{j \in J} N_{j}$ and $P / L$ has a composition series. This contradicts the maximality of $K$ in $A_{r}(R / M, R)$. Since $\left\{y_{j}\right\}_{j \in J}$ is a basis for $\mathcal{O},\left(y_{j}\right)^{\perp} \neq\left(y_{h}\right)^{\perp}$ if $j \neq h$. Well-order $J$ beginning the order with the positive integers.

Let $e=e^{2} \in\left(y_{1}\right)^{\perp}, e \notin\left(y_{2}\right)^{\perp}$. By Lemma 2.1, $(1-e)+\left(y_{j}\right)^{\perp} \neq 0$ for an infinite set $H \subseteq J$.

Assume that $y_{h}(1-e) N_{1} \subseteq\left(y_{h}\right)^{\perp}$ for all but' a finite number of $h \in H$, say $\left\{h_{1}, h_{2}, \ldots, h_{n}\right\}$. Let $H^{\prime}=H-\left\{h_{1}, \ldots, h_{n}\right\}$. Let $V=\bigoplus_{h \in H^{\prime}} F y_{h}$. For all $h \in H^{\prime}$, $y_{h}(1-e)=f_{h}\left(y_{1}(1-e)\right)$ for some $f_{h} \in F$ and all $h \in H^{\prime}$. Since $y_{1}(1-e) \in V$, $\left\{y_{1}(1-e)\right\} \cup\left\{y_{h} e\right\}_{h \in H}$ is a generating set for $V$. A basis containing $y_{1}(1-e)$ may be picked out, say $\left\{y_{1}(1-e)\right\} \cup\left\{y_{h} e\right\}_{h \in G}$ where $G \subseteq H^{\prime}$. Then $1-e \in\left(y_{h} e\right)^{\perp}$ for all $h \in G$. Let $Q_{h}=\left\{y_{h} e\right\}^{\perp}$ for all $h \in H$. Then $Q=\bigcap_{h \in G} Q_{h} \supset_{+} K$ and $Q / K$ has a composition series, since $K=Q \cap\left(y_{1}(1-e)\right)^{\perp}$. Therefore $y_{h}(1-e) N_{1} \ddagger\left(y_{h}\right)^{\perp}$ for an infinite number of $h$ and the hypothesis of Lemma 2.3 hold. Thus $E(R / M)$ is not $\Sigma$-injective.

THEOREM 2.6. Let $R$ be a regular ring. Let $T=\bigoplus_{i \in I} E\left(R / M_{i}\right)$. Let $H=\left\{R / M_{i}\right\}_{i \in I}$. Then $T=E(T)$ iff $H$ contains only a finite number of nonisomorphic simples, and if $R / M_{j}$ is any simple in $H$, either it is finite dimensional over its endomorphism ring or $H$ contains only a finite number of isomorphic copies of $i t$.

Proof. If $T=E(T)$ the result follows from Theorem 2.5 and Proposition 2.4 and the fact that direct summands of injective modules are injective.

The converse is trivial.

Tiwary [13] has shown that if $R$ is a commutative regular ring, then every simple module is $\Sigma$-injective. This follows from our result since in this case every simple $R$-module is one-dimensional over its endomorphism ring.

3. The direct product as injective hull. We will now look at the other extreme, when does $E(T)=\prod_{i \in I} E\left(R / M_{i}\right)$ ?

Proposition 3.1. Let $R$ be a ring such that every cyclic $R$-module contains a simple $R$-module. If $\left\{M_{i}\right\}_{i \in I}$ is any collection of maximal right ideals, such that $R / M_{i} \not \approx R / M_{j}$ for $i \neq j$, then $E\left(\oplus_{i \in I} E\left(R / M_{i}\right)\right)=\prod_{i \in I} E\left(R / M_{i}\right)$.

Proof. Let $W=\prod_{i \in I} E\left(R / M_{i}\right)$. Let $0 \neq x=\left(x_{i}\right)_{i \in I} \in W$ be arbitrary. We will show that $x R \cap T \neq 0$, where $T=\bigoplus_{i \in I} E\left(R / M_{i}\right)$. Thus $W$ will be an injective essential extension of $T$ and therefore $W=E(T)$.

Let $L=\bigcap_{i \in I}\left(X_{i}\right)^{\perp}$. Let $K / L \subseteq R / L$ be a simple $R$-module, where $K$ is a right ideal. We claim $K \subseteq \bigcap_{i \neq j}\left(X_{i}\right)^{\perp}$ for some $j \in I$. Let $k+L$ generate $K / L$. Then $k \notin\left(X_{j}\right)^{\perp}$ for some $j \in I$. If $k \notin\left(X_{t}\right)^{\perp}, t \neq j, t \in I$, then $k+\left(X_{j}\right)^{\perp} \neq 0+\left(X_{j}\right)^{\perp}$ and $k+\left(X_{i}\right)^{\perp} \neq 0+\left(X_{t}\right)^{\perp}$.

Now no nonzero submodule of $x_{t} R$ is isomorphic to a submodule of $x_{j} R$, since they are essential extensions of nonisomorphic socles. Hence

$$
\left(k+\left(X_{t}\right)^{\perp}\right)^{\perp} \neq\left(k+\left(X_{j}\right)^{\perp}\right)^{\perp} .
$$


Without loss of generality there exists an $r \in\left(k+\left(X_{j}\right)^{\perp}\right)^{\perp}, r \notin\left(k+\left(X_{t}\right)^{\perp}\right)^{\perp}$ since we can always interchange the roles of $j$ and $t$. Then $k r \in\left(X_{j}\right)^{\perp}, k r \notin\left(X_{t}\right)^{\perp}$. Therefore $k r+L \neq 0+L$. Since $K / L$ is simple, there exists $m \in L$ and $r^{\prime} \in R$ such that $k=k r r^{\prime}+m$. Since $k r \in\left(X_{j}\right)^{\perp}$ and $L \subseteq\left(X_{j}\right)^{\perp}, k \in\left(X_{j}\right)^{\perp}$, a contradiction. Therefore $K \subseteq \bigcap_{i \neq j}\left(X_{i}\right)^{\perp}$. Then $0 \neq x k \in T$. Thus $E(T)=W$.

It might seem that Proposition 3.1 comes from nowhere, but in the commutative regular case this is also a necessary condition.

THEOREM 3.2. Let $R$ be a commutative regular ring. Let $T=\bigoplus_{i \in I} R / M_{i}$, where $\left\{M_{i}\right\}_{i \in I}$ is any set of distinct maximal ideals. Then $\prod_{i \in I} R / M_{i}=E\left(\oplus_{i \in I} R / M_{i}\right)$ for all such collections $\left\{M_{i}\right\}_{i \in I}$ iff every cyclic $R$-module contains a simple $R$-module.

Proof. If every cyclic $R$-module contains a simple $R$-module, then this is Proposition 3.1.

Assume $E\left(\oplus_{i \in I} R / M_{i}\right)=\prod_{i \in I}\left(R / M_{i}\right)$ for all collections $\left\{M_{i}\right\}_{i \in I}$ of distinct maximal ideals. Let $P$ be a cyclic $R$-module. Then $P \approx R / L$ where $L$ is an ideal. Since $R$ is a $V$-ring, $L=\bigcap_{i \in I} M_{i}$ where each $M_{i}$ is a maximal ideal.

Let $x=\left(x_{i}\right)_{i \in I} \in \prod_{i \in I} R / M_{i}, x_{i} \neq 0$ for all $i \in I$. Since $E\left(\oplus_{i \in I} R / M_{i}\right)=\prod_{i \in I} R / M_{i}$, there exists an $r \in R$ such that $0 \neq x r \in T$. In fact $r$ can be picked in all but one $M_{k}$ for some $k \in I$. Thus $0 \neq x r \in R / M_{k}$.

Set $K=\bigcap_{i \neq k} M_{i} \supset \bigcap_{i \in I} M_{i}$. Thus $K / L$ is simple and the theorem is proved.

4. Irredundant intersections of maximal ideals. Another interesting problem that arises from this work is the irredundancy of intersections of right ideals.

In Proposition 3.1 and Theorem 3.2 we investigated when the injective hull of $T=\oplus_{i \in I} E\left(R / M_{i}\right)$ is equal to $W=\prod_{i \in I} E\left(R / M_{i}\right)$.

We found that a sufficient condition is that every cyclic $R$-module contains a simple $R$-module. In the commutative regular case if $L=\bigcap_{i \in I} M_{i}$ where $\left\{M_{i}\right\}_{i \in I}$ is any set of maximal ideals, then $R / L \supseteq K / L$ where $K$ is an ideal and $K / L$ is simple.

The method used in the proof of Proposition 3.1 shows $K=\bigcap_{i \neq j} M_{i}$ for some $j \in I$. Can one write $L$ as an irredundant intersection of maximal ideals?

The sufficient condition of Nastasescu and Popescu [8], that every cyclic $R$ module contains a simple $R$-module, is too strong to be necessary. The important cyclics for this are of the form $R / \bigcap_{i \in I} M_{i}$, where $\left\{M_{i}\right\}_{i \in I}$ is any collection of maximal ideals.

THEOREM 4.1. Let $R$ be a commutative ring. Then every intersection of maximal ideals can be written as an irredundant intersection of maximal ideals iff every cyclic $R$-module of the form $R / \bigcap_{i \in I} M_{i}$, where $\left\{M_{i}\right\}_{i \in I}$ represents any set of maximal ideals, contains a simple $R$-module.

Proof. Assume that every intersection of maximal ideals can be written as an irredundant intersection of maximal ideals. Let $\left\{M_{i}\right\}_{i \in I}$ be any collection of maximal ideals. Let $L=\bigcap_{i \in I} M_{i}=\bigcap_{j \in J} N_{j}$ where $\left\{N_{j}\right\}_{j \in J}$ is a collection of maximal ideals such that their intersection is irredundant. Then $L \subseteq \bigcap_{j \neq k} N_{j}$ for any $k \in J$, since the 
intersection is irredundant. Let $K=\bigcap_{j \neq k} N_{j}$ for some $k \in I . K / L \approx R / N_{k}$ is simple. Therefore every cyclic of the required form contains a simple module.

Assume that every cyclic module of the required form contains a simple module. Let $\bigcap_{i \in I} M_{i}$ be any intersection of maximal ideals. We will show that $L=\bigcap_{i \in I} M_{i}$ can be written as an irredundant intersection of maximal ideals.

Since $R=R / \bigcap_{i \in I} M_{i}$ is cyclic of the required form, $R$ contains a simple $R$-module and thus $R$ has nonzero scale.

By Proposition 3.1 we know that every simple $R$-submodule of $R$ is the intersection of all but one maximal ideal in $\left\{M_{i}\right\}_{i \in I}$. Let $\left\{S_{j / L}\right\}_{j \in J}$ be the set of distinct simple submodules of $R$, where $J \subseteq I$. That is $S_{j}=\bigcap_{i \neq j} M_{i}$. Let $H=\bigcap_{j \in J} M_{j}$.

We claim $H=L$ and that $H$ is an irredundant intersection. It is obvious that $H \supseteq L$. If it were proper containment let $a \in H, a \notin L . H / L$ has zero socle, since if it contained a simple submodule $S, S$ would equal $\bigcap_{i \neq j} M_{i}$ for some $j \in J$. This is impossible since $\bigcap_{i \neq j} M_{i} \nsubseteq M_{j}$ since $\bigcap_{i \neq j} M_{i} \neq L$.

We will show that $a R+L / L$ contains a simple $R$-module and thus arrive at a contradiction. Consider the collection $\left\{M_{t}\right\}_{t \in T}$ where $T \subseteq I$ and $a \notin M_{t}$ for all $t \in T$. We claim $a R+L / L \approx R / \bigcap_{t \in T} M_{t}$. By the hypothesis this latter cyclic contains a simple $R$-module.

To get this isomorphism define a map $f: R / \bigcap_{t \in T} M_{t} \rightarrow a R+L / L$ by $f\left(r+\bigcap_{t \in T} M_{t}\right)$ $=a r+L$. Then $f$ is well defined since if $r \in \bigcap_{t \in T} M_{t}$, ar $\in L \cdot f$ is clearly onto. $f\left(r+\bigcap_{t \in T} M_{t}\right)=0+L$ implies $a r \in L$ since $a \notin M_{t}$ for all $t \in T, r \in M_{t}$ for all $t \in T$, since each $M_{t}$ is prime. Thus we have the isomorphism and $H=L$.

If $H$ is not the irredundant intersection of the $\left\{M_{j}\right\}_{j \in J}$ then $\bigcap_{j \neq k} M_{j}=\bigcap_{j \in J} M_{j}$ for some $k \in J$. This would say that $M_{k} \supseteq \bigcap_{j \neq k} M_{j}$, but the $\left\{M_{j}\right\}_{j \in J}$ were picked so this could not happen. Therefore $L$ is an irredundant intersection of maximal ideals.

An example of a ring with this property which is not regular is $R=\bigoplus_{i=1}^{\infty} Q_{i}+Z_{(2)}$, where each $Q_{i}$ is a copy of the rational numbers and $Z_{(2)}$ is the integers localized at 2Z. $R$ consists of elements of the form $r=\left(q_{1}, q_{2}, \ldots, q_{n}, a_{1} a_{1} a_{1} \ldots\right)$, where each $q_{i} \in Q$ and $a \in Z_{(2 ;} \subseteq Q$.

5. The Zariski topology. Let $R$ be a ring such that every one-sided ideal is twosided. Let spec $R$ be the set of all maximal ideals of $R$. Let $\mathcal{O}$ be the collection of all sets of the form

$$
H(a)=\{M \in \operatorname{spec} R \mid a \notin M\}
$$

for all $a \in R$. Then $\mathcal{O}$ is a basis for a compact topology on $\operatorname{spec} R$ [7, p. 47]. For topological notation see Kelley [5]. This topology is called the Zariski topology on spec $R$.

If $R$ is also regular, then the Zariski topology is Hausdorff. To see this let $M_{1}, M_{2} \in \operatorname{spec} R$. Let $e=e^{2} \in M_{1}, e \notin M_{2}$. Then $M \in H(1-e)$ and $M_{2} \in H(e)$, and these are disjoint open sets.

If $R$ is regular then each $H(a)$ is both open and closed. Let $a R=e R$ where $e=e^{2}$. Then $H(a)=H(e)$ and $e$ belongs to a maximal ideal $M$ iff $1-e \notin M$. 
In the special case when $R=\prod_{i \in I} F_{i}$, where each $F_{i}$ is a division ring, it is known that the Zariski topology on spec $R$ is homeomorphic to the Stone-Čech compactification of the set $I$ with the discrete topology.

We will now look at the closure of sets in $\operatorname{spec} R$.

Proposition 5.1. Let $R$ be a regular ring such that every right ideal is two-sided. Let $C=\left\{M_{j}\right\}_{j \in J}$ be a subset of $\operatorname{spec} R$. Then the closure of $C$ consists of all those maximal ideals $M$ such that $M \supseteq \bigcap_{j \in J} M_{j}$.

Proof. Let $M$ belong to the closure of $C$. Let $e \in \bigcap_{i \in I} M_{i}$, where $e=e^{2}$. If $e \notin M$, then $1-e \in M$. Since $H(e)$ is a neighborhood of $M$ and $M$ is a point of closure of $C, M_{j} \in H(e)$ for some $j \in J$. Thus $e \notin M_{j}$, a contradiction. Therefore $M \supseteq \bigcap_{j \in J} M_{j}$.

Let $M \supseteq \bigcap_{j \in J} M_{j}$. Let $H(1-e)$ be a basic neighborhood of $M$. Assume $M_{j} \notin H(1-e)$ for all $j$. Then $1-e \in M_{j}$ for all $j$ so $1-e \in \bigcap_{j \in J} M_{j} \subseteq M$, a contradiction. Therefore $M$ belongs to the closure of $C$.

PROPOSITION 5.2. Let $R$ be a regular ring such that every right ideal is two-sided. Let $C$ be any closed set and $M \notin C$. Then there is a basic open set, $H(e)$, such that $H(e) \supseteq C$ and $M \notin H(e)$, where $e=e^{2}$.

Proof. $C=\left\{M_{j}\right\}_{j \in J}$ for some set $J$. Since $M$ is not in the closure of $C$, $M \ddagger_{+} \bigcap_{j \in J} M_{j}$. Let $e=e^{2} \in \bigcap_{j \in J} M_{j}, e \notin M$. Then $H(e)$ is the desired set.

This proposition shows that if $C$ is any closed set and $M \notin C$, there exist two open sets $\mathrm{P}_{1}, \mathrm{P}_{2}$ such that $M \in \mathrm{P}_{1}, C \subseteq \mathrm{P}_{2}$ and $\mathrm{P}_{1} \cup \mathrm{P}_{2}=\operatorname{spec} R$. Let $\mathrm{P}_{2}=H(e)$ and $\mathrm{P}_{1}=H(1-e)$ as in Proposition 5.2.

Let $R$ be a commutative regular ring. Let $T=E\left(\oplus_{i \in I} R / M_{i}\right)$ where $\left\{M_{i}\right\}_{i \in I}$ is any collection of maximal ideals. Tiwary [3] has shown that, if $T$ is injective, then $\left\{M_{i}\right\}_{i \in I}$ contains only a finite number of isolated points in the relative topology on $\left\{M_{i}\right\}_{i \in I}$. We will give an algebraic description of all the isolated points in spec $R$, where $R$ is any ring such that every right ideal is two-sided. Let $L=\bigcap_{i \in I} M_{i}$ where $\left\{M_{i}\right\}_{i \in I}$ is the set of all maximal ideals of $R$. Let $K_{j}=\bigcap_{i \neq j} M_{i}$ for all $j \in I$.

Proposition 5.3. Let $R$ be a ring with all right ideals two-sided. The following are equivalent:

(i) $K_{j} / L$ is simple.

(ii) $M_{j}$ is an isolated point in spec $R$.

Proof. Assume (i). Let $e \in K_{j}, e \notin M_{j}$. Then $H(e)=\left\{M_{j}\right\}$. Therefore $M_{j}$ is an isolated point.

Now assume (ii). Let $H(e)=\left\{M_{j}\right\}$. Then $e \in K_{j}, e \notin M_{j}$. Therefore $K_{j} / L$ is simple.

Alexandroff and Urysohn have shown that if $|I|$ is countable, then $B(I)$ has the property that the only converging sequences are those that are constant from some point on [1]. Their proof also goes through for any set $I$. We will give an algebraic proof of this fact and show that any complete regular ring whose idempotents are central has this property. By a regular ring $R$ being complete we mean the lattice of 
cyclic right ideals of $R$ form a complete lattice. Any direct product of divisions rings is a complete regular ring.

LemMA 5.4. Let $R$ be a regular ring whose idempotents are central. Let $\left\{M_{j}\right\}_{j \in J}$ be a collection of maximal ideals. Let $L=\bigcap_{j \in J} M_{j}$. Then idempotents in $R / L$ can be lifted to $R$. Furthermore countable sets of orthogonal idempotents in $R / L$ can be lifted to a set of orthogonal idempotents in $R$.

Proof. The proof is obvious. If $a+L=(a+L)^{2}$, then $a+L$ lifts to $e$ where $e R=a R$ and $e^{2}=e$.

A family $B=\{e(j) \mid j \in J\}$ of idempotents of a ring $R$ is called nice if

(i) $e(i) e(j)=e(j) e(i)$ for all $i, j \in J$,

(ii) $\prod_{\alpha=1}^{n}\left(j_{\alpha}\right) \prod_{\beta=n+1}^{m}\left(1-e\left(j_{\beta}\right)\right) \neq 0$ if $\left\{j_{\alpha} \mid 1 \leqq \alpha \leqq m\right\} \cap\left\{j_{\beta} \mid n+1 \leqq \beta \leqq m\right\}=\varnothing$.

The following proposition follows from a result of Hausdorff and Tarski on independent elements in the Boolean algebra of subsets of a set.

Proposition 5.5. (See Sikorski [12, p. 45], Osofsky [9].) Let $\{\varepsilon(k) \mid k \in K\}$ be an infinite set of orthogonal idempotents of a ring $R$ with $|K|=\aleph$. Assume for each $L \subseteq K$ there exists an idempotent $e(L) \in R$ such that

(i) $\varepsilon(k) e(L)=e(L) \varepsilon(k)=\varepsilon(k) X_{L}(k)$ where $X_{L}$ denotes the characteristic function of $L$.

(ii) $e(L) e(H)=e(H) e(L)$ for all $L, H \subseteq K$.

Then there exists a nice set of idempotents $A \subseteq R$ such that $|A|=2^{\aleph}$. Also if $\left\{a_{j_{1}}, \ldots, a_{j_{n}}\right\} \subseteq A$, there exists $k \in K$ such that $\varepsilon(k) \prod_{\alpha=1}^{n} a\left(j_{\alpha}\right) \prod_{\beta=n+1}^{m}\left(1-a\left(j_{\beta}\right)\right)$ $=\varepsilon(k)$.

Proposition 5.6. (See Osofsky [9].) Let $R$ be a complete regular ring. For any infinite set of orthogonal idempotents of $R$ the hypotheses of Proposition 5.5 are satisfied.

THEOREM 5.7. Let $R$ be a complete regular ring such that all idempotents are central. Let $\left\{M_{j}\right\}_{j=1}^{\infty}$ be a set of distinct maximal ideals. Then $M_{1}, \ldots, M_{n}, \ldots$ does not converge in the Zariski topology.

Proof. Assume that $M_{1}, \ldots, M_{n}, \ldots$ converges to $M$. Let $f \in M$. Then $f$ belongs to all but a finite number of the $M_{j}$ 's. Therefore $M \subseteq \bigcup_{n}\left(\bigcap_{j \geqq_{n}} M_{j}\right)$. Since this latter ideal is proper $M=\bigcup_{n}\left(\bigcap_{j \geqq n} M_{j}\right)$. Let $L=\bigcap_{j=1}^{\infty} M_{j}$. By the methods of Proposition 3.1 $M / L$ is the socle of $R / L$.

Let $N_{k}=\bigcap_{j \neq k} M_{j}$. Then every simple submodule of $R / L$ must be of this form. If the sequence converges there are only a countable number of maximal ideals containing $L$, since for every maximal ideal $N \neq M$ containing $L, N / L$ is a direct summand of $R / L$. This follows since $M / L$ is the socle of $R / L$ and $N$ misses some idempotent generated simple submodule of the socle.

Let $\left(a_{k}+L\right)=\left(a_{k}+L\right)^{2}$ generate $N_{k}$ for all $k$. By Lemma 5.4 lift these elements to an orthogonal set $\left\{e_{k}\right\}_{k=1}^{\infty}$ of idempotents in $R$. Now use Proposition 5.5 and 
Proposition 5.6 to construct a nice set of idempotents in $R$ of cardinality $2^{\aleph_{0}}$. Also by Proposition 5.5 these elements $\bmod L$ will form a nice set of idempotents, $B$, of cardinality $2^{N_{0}}$. For each $f \in 2^{B}$ construct an ideal $I_{f}$ of $R / L$ by admitting $e$ if $f_{e}=1$ or $1-e$ if $f_{e}=0$. Enlarge each $I_{f}$ to a maximal ideal $M_{f}$ of $R / L$. These maximal ideals are distinct since $f \neq g$ implies there exist $e \in M_{f}$ such that $1-e \in M_{g}$. This shows that there are at least $2^{2 \text { No }_{0}}$ of these maximal ideals. Thus the sequence does not converge.

This theorem does not hold in arbitrary commutative regular rings. Let $R=\oplus_{i=1}^{\infty} F_{i}+F$ where $F$ is a field and each $F_{i}=F$. Elements of $R$ are of the form $\left(f_{1}, \ldots, f_{n}, f, f, f, \ldots\right)$ where each $f_{i} \in F$ and $f \in F$. Let $M_{i}$ be the maximal ideal with $i$ th component zero. Let $M=\bigoplus_{i=1}^{\infty} F_{i}$ then $M$ is maximal and $M_{1}, \ldots, M_{n} \ldots$ converges to $M$.

Let $L=\bigcap_{i=1}^{\infty} M_{i}$. In this case $M / L$ is the socle of $R / L$. This characterizes those sequences that converge.

THEOREM 5.8. Let $R$ be a regular ring whose idempotents are central. Let $M_{1}, \ldots, M_{n}, \ldots$ be a sequence of distinct maximal ideals. Let $L=\bigcap_{i=1}^{\infty} M_{i}$. Then $M_{1}, \ldots, M_{n} \ldots$ converges to a maximal ideal $M$ iff $M / L$ is the socle of $R / L$. In this case $E\left(\oplus_{i \in I} R / M_{i}\right)=\prod_{i \in I} R / M_{i}$.

Proof. Assume that $M_{1}, \ldots, M_{n}$.. converges to $M$. Then as in Theorem 5.7 $M=\bigcup_{n}\left(\bigcap_{i \geqq n} M_{i}\right)$ and $M / L$ is the socle of $R / L$.

Now assume that $M / L$ is the socle of $R / L$. Let $f \in M$. The socle of $R / L$ is $\sum K_{i}$ where $K_{j}=\bigcap_{i \neq j} M_{i} / L$ for each $j$. Thus $f$ belongs to all but a finite number of $\left\{M_{i}\right\}_{i=1}^{\infty}$. Therefore $M_{1}, \ldots, M_{n}, \ldots$ converges to $M$.

Let $0 \neq x=\left(x_{i}\right)_{i=1} \in \prod_{i=1}^{\infty} R / M_{i}$. Let $J=\left\{i \mid x_{i} \neq 0\right\}$. If $J$ is infinite $\left\{M_{j}\right\}_{j \in J}$ is a subsequence of $\left\{M_{i}\right\}_{i=1}^{\infty}$ and thus converges to $M$. Therefore there exists an $r \in R$ such that $r$ belongs to all but a finite number of the $\left\{M_{j}\right\}_{j \in J}$. Then $0 \neq x r \in \bigoplus_{i=1}^{\infty} R / M_{i}$, so $\prod_{i=1}^{\infty} R / M_{i}$ is an injective essential extension of $\sum_{i=1}^{\infty} R / M_{i}$. Thus $E\left(\oplus_{i=1}^{\infty} R / M_{i}\right)=\prod_{i=1}^{\infty} R / M_{i}$.

\section{REFERENCES}

1. E. Cech, On bicompact spaces, Ann. of Math. (2) 38 (1937), 823-844.

2. B. Eckmann and A. Schopf, Über injektive Moduln, Arch. Math. 4 (1953), 75-78. MR 15, 5.

3. C. Faith, Rings with ascending condition on annihilators, Nagoya Math. J. 27 (1966), 179191. MR 33 \#1328.

4. R. E. Johnson and E. T. Wong, Quasi-injective modules and irreducible rings, J. London Math. Soc. 36 (1961), 260-268. MR 24 \#A1295.

5. J. L. Kelley, General topology, Van Nostrand, Princeton, N. J., New York, 1955. MR 16, 1136.

6. S. Kochen, Ultraproducts in the theory of models, Ann. of Math. (2) 74 (1961), 221-261. MR 25 \#1992.

7. J. Lambek, Lectures on rings and modules, Blaisdell, Waltham, Mass., 1955.

8. C. Năstăsescu and N. Popescu, Anneaux semi-artiniens, Bull. Soc. Math. France 96 (1968), 357-368. MR 39 \#247. 
9. B. L. Osofsky, Homological dimension and cardinality, Trans. Amer. Math. Soc. 151 (1970), 641-649.

10. A. Rosenberg and D. Zelinski, Finiteness of the injective hull, Math. Z. 70 (1958/59), 372-380. MR 21 \#4176.

11. W. Rudin, Homogeneity problems in the theory of Cech compactifications, Duke Math. J. 23 (1956), 409-419, 633. MR 18, 324.

12. R. Sikorski, Boolean algebras, 3rd ed., Ergebnisse der Mathematik und ihrer Grenzgebiete, Band 25, Springer-Verlag, New York, 1969. MR 39 \#4053.

13. A. K. Tiwary, Injective hulls of semi-simple modules over regular rings, Pacific J. Math. 31 (1969), 247-254.

14. J. von Neumann, On regular rings, Proc. Nat. Acad. Sci. U.S.A. 22 (1936), 707-713.

\author{
Rutgers-The State University, \\ New BRunswick, New Jersey 08903 \\ Monmouth College, \\ West LoNg Branch, New Jersey 07764
}

